\title{
Initial stage sintering of polymer particles - Experiments and modelling of size-, temperature- and time-dependent contacts
}

\author{
Regina Fuchs ${ }^{1, \star}$, Thomas Weinhart ${ }^{2, \star \star}$, Ming Ye ${ }^{1}$, Stefan Luding ${ }^{2}$, Hans-Juergen Butt ${ }^{1}$, and Michael Kappl ${ }^{1, \star \star \star ~}$ \\ ${ }^{1}$ Max-Planck-Institute for Polymer Research, Physics at Interfaces, Ackermannweg 10, 55218 Mainz, Germany \\ ${ }^{2}$ Multiscale Mechanics, Engineering Technology, MESA+, University of Twente, PO Box 217, 7500 AE Enschede, The Netherlands
}

\begin{abstract}
.
The early-stage sintering of thin layers of micron-sized polystyrene (PS) particles, at sintering temperatures near and above the glass transition temperature $T_{\mathrm{g}}\left(\sim 100^{\circ} \mathrm{C}\right)$, is studied utilizing $3 \mathrm{D}$ tomography, nanoindentation and confocal microscopy. Our experimental results confirm the existence of a critical particle radius $\left(r_{\text {crit }} \sim\right.$ $1 \mu \mathrm{m}$ ) below which surface forces need to be considered as additional driving force, on top of the usual surfacetension driven viscous flow sintering mechanism. Both sintering kinetics and mechanical properties of particles smaller than $r_{\text {crit }}$ are dominated by contact deformation due to surface forces, so that sintering of larger particles is generally characterized by viscous flow. Consequently, smaller particles require shorter sintering. These experimental observations are supported by discrete particle simulations that are based on analytical models: for small particles, if only viscous sintering is considered, the model under-predicts the neck radius during early stage sintering, which confirms the need for an additional driving mechanism like elastic-plastic repulsion and surface forces that are both added to the DEM model.
\end{abstract}

\section{Introduction}

Recent developments in 3D printing and Additive Manufacturing [1] enable the fabrication of individualized serial products based on powders and grains. To date, none of the conventional techniques like, e.g., selective laser sintering allow the production of interconnected porous polymer scaffolds with a variety of pore sizes and reproducible morphology, which are commonly used to stimulate the formation of new tissue [2]. This forces the attention of scientists to the initial stage of sintering, where the porous structure is characterized by the formation of necks between individual particles. Achieving the desired end-product by initial stage sintering of polymer particles of various sizes without a significant temperature increase in the bulk of each particle requires a fundamental understanding of sintering under varying temperature-, pressure- and time-conditions. In the present work, initial stage sintering of polystyrene (PS) particle layers $(<30$ particle diameters) with radii of $0.25-2 \mu \mathrm{m}$ was analysed utilizing 3D tomography (FIB/SEM), nanoindentation and confocal microscopy. The mechanical properties as well as the real-time topography deformation within the sintered particle layers during nanoindentation are investigated. Moreover, the experimental results are correlated with Discrete Element Method (DEM) simulations to calibrate a temperature- and pressure-dependent sintering model that includes the contribution of surface forces.

\footnotetext{
^e-mail: fuchsr@mpip-mainz.mpg.de

$\star \star$ e-mail: t.weinhart@utwente.nl

$\star \star \star$ e-mail: kappl@mpip-mainz.mpg.de
}

\section{Experimental details}

\subsection{Preparation of particle and samples}

PS spheres featuring nominal particle radii of $0.25 \mu \mathrm{m}$, $0.5 \mu \mathrm{m}, 0.75 \mu \mathrm{m}$ and $2 \mu \mathrm{m}$, molecular weights $\left(M_{w}\right)$ of $110709-312010 \mathrm{~g} / \mathrm{mol}$ and a glass transition temperature $T_{\mathrm{g}}$ of $94-99^{\circ} \mathrm{C}$ were synthesized by dispersion polymerization as reported in Zhang et al. [3] and stored in aqueous solution. For each particle radius, multi-layer films were realized by placing $10 \mu \mathrm{l}$ of a particle dispersion $(1: 1$ volume ratio, PS particle:ethanol) on an oxygen-plasma hydrophilised glass substrate and dried for at least $24 \mathrm{~h}$ in air. Sintering was carried out in air on a heating stage at temperatures of $90^{\circ}, 95^{\circ}, 100^{\circ}, 105^{\circ}$ and $110^{\circ} \mathrm{C}$. The rate of temperature change was set to be $15^{\circ} \mathrm{C} / \mathrm{min}$ during heating. Holding periods of 20, 40, 60, 90, 120 and 180 s were set at each temperature for each particle radius, respectively. After sintering, the samples were quickly cooled down to room temperature on a metal platform. The average layer thickness was reduced by up to max. $50 \%$ as the sintering temperature increases from room temperature to $110^{\circ} \mathrm{C}$. Fluorescently labeled PS particles with radii of $2 \mu \mathrm{m}$ were prepared by staining the PS shell of the particles with a solution of Nile Red and Xylol for $24 \mathrm{~h}$. Afterwards, the solvent was removed under vacuum.

\subsection{Characterization of microstructure with FIB/SEM reconstruction}

The sintered PS particle films were coated with $100 \mathrm{~nm}$ Pt layer on top. A focused-ion beam instrument com- 
bined with a scanning electron microscope (FIB/SEM), (Nova 600 Nanolab, FEI, USA), was used for sectioning and imaging sequential 2D cross-sectional surface images. A protective Pt layer of $1 \mu \mathrm{m}$ thickness and $12 \times$ $8 \mu \mathrm{m}^{2}$ area was deposited on the sample. A volume of $12 \times 8 \times 6 \mu \mathrm{m}^{3}$ was milled slice-by-slice with $20 \mathrm{~nm}$ distance between two consecutive images. The actual 3D volumes of the sintered films were reconstructed based on the as-recorded stacks of images using Amira 4.1 (Visage Imaging, San Diego, USA). The reconstruction process involved i) alignment of the images, ii) resampling and iii) segmentation. The radius of at least 12 particles as well as the mean neck radius $x$ was determined for each sintered sample.

\subsection{Characterization of mechanical properties with Nanoindentation and Confocal Microscopy}

Nanoindentation measurements were performed with a MFP NanoIndenter (Asylum Research, Santa Barbara, CA, spring constant $k=2390 \mathrm{~N} / \mathrm{m}$ ) equipped with a spherical ruby indenter $(d=127 \mu \mathrm{m})$. Indentations were performed in load-controlled mode. The applied load varies between 1 and $4 \mathrm{mN}$ with loading rates between $200 \mu \mathrm{N} / \mathrm{s}$ and $800 \mu \mathrm{N} / \mathrm{s}$. In order to get an estimate of the standard deviation of the mechanical properties, each sample was tested at least at 12 individual positions separated by at least $100 \mu \mathrm{m}$. The reduced elastic modulus $\left(E_{\text {red }}\right)$ for each sample was obtained from the unloading portion of the load-displacement curve using the Oliver and Pharr method with a spherical area function [4]. Additionally, the nanoindenter was placed on the sample stage of a custom-built laser scanning confocal microscope (LSCM), which has the capability to measure insitu the real-time deformation within the sintered particle layers during nanoindentation. The scan rate of the LSCM was one 2D frame per second with a sampling resolution of $0.235 \mu \mathrm{m} /$ pixel in $\mathrm{x}-$ and $\mathrm{y}$-direction and $0.570 \mu \mathrm{m} /$ pixel in $\mathrm{z}$-direction. The ruby sphere was indented into the sintered fluorescently labelled PS particle film in displacement-controlled mode with a maximum displacement of $5 \mu \mathrm{m}$, while the structure was imaged by LSCM. For the LSCM imaging, the sintered films needed to be infiltrated with a liquid that matches the refractive index of the PS (Cargille Laboratories, USA).

\section{Contact mechanics}

To simulate the sinter and indenting process, we model adhesive, elastoplastic, and dissipative contact forces, with the normal force between two overlapping spherical particles given by

$$
f_{\mathrm{n}}=-f_{\mathrm{a}}+k\left(\delta-\delta_{\mathrm{p}}\right)+\gamma \dot{\delta} \quad \text { if } \quad \delta>\delta_{\mathrm{p}} .
$$

where $\delta$ denotes the total particle overlap and $\delta_{\mathrm{p}}$ the plastic deformation. The adhesion force $f_{\mathrm{a}}$ is assumed constant, while the elastoplastic and dissipative forces are linear functions of the effective, elastic overlap $\left(\delta-\delta_{\mathrm{p}}\right)$ and normal velocity $\dot{\delta}$, respectively, with $k$ denoting the contact stiffness and $\gamma$ the dissipation coefficient, see also [5].

We further assume that the stiffness increases with the amount of plastic deformation, due to the increased contact surface, with $k=k_{1}$ for zero plastic overlap up to a maximum of $k=k_{\text {crit }}$ at maximum plastic overlap $\delta_{\mathrm{p}}^{\text {crit }}$, which is set to avoid solid volume fractions above one (fully sintered) [5]:

$$
k=k_{1}+\left(k_{\text {crit }}-k_{1}\right)\left(\delta_{\mathrm{p}} / \delta_{\mathrm{p}}^{\text {crit }}\right)^{\psi} .
$$

The power $\psi$ can be set to $\psi=1 / 2$ to reproduce Hertz-like behavior, or to any other value including $\psi=0$ to deactivate this non-linearity, or to a linear interpolation with $\psi=1$, as done in the following. Below $\delta_{\mathrm{p}}=\delta_{\mathrm{p}}^{\text {crit }}$, particles are soft, and thus deform, proportionally to the overlap, when the overlap between the particles reaches a new maximum (i.e. $\left.k\left(\delta-\delta_{\mathrm{p}}\right)>k_{1} \delta\right)$.

$$
\delta_{\mathrm{p}}= \begin{cases}\frac{k-k_{1}}{k} \delta & \text { if } k\left(\delta-\delta_{\mathrm{p}}\right)>k_{\mathrm{l}} \delta . \\ \frac{k+k_{\mathrm{c}}}{k} \delta & \text { if } k\left(\delta-\delta_{\mathrm{p}}\right)<-k_{\mathrm{c}} \delta .\end{cases}
$$

The reduction in plastic overlap during unloading is introduced to model contact cohesion. This causes the loadingunloading-reloading behavior shown in Figure 1.

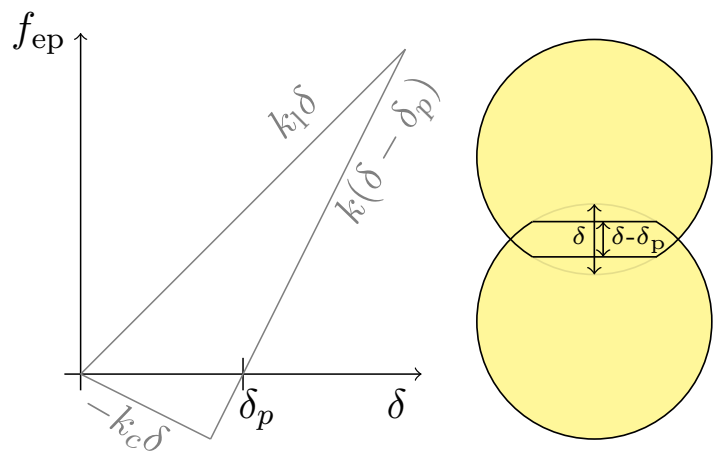

Figure 1. Elasto-plastic contact law.

All parameters of the contact model - and the particle density - may vary with temperature [6], but we neglect this effect here for simplicity, assuming constant values. At temperatures above the glass transition, viscous flow causes the particles to sinter, which creates an increase in plastic overlap, modelled here as

$$
\dot{\delta}_{\mathrm{p}}=\frac{f_{\mathrm{n}} r_{\mathrm{p}}}{n f_{\mathrm{a}} \tau_{\mathrm{s}}}\left(\frac{r_{\mathrm{p}}}{x}\right)^{n-1} .
$$

For two particles without compression $\left(f_{\mathrm{n}}=f_{a}\right)$ and of high stiffness $\left(\sqrt{m / k} \ll \tau_{\mathrm{s}}\right)$, we can assume that $\delta=\delta_{\mathrm{p}}$, therefore the plastic overlap increases as $\delta_{\mathrm{p}} / r_{\mathrm{p}}=\sqrt[n]{t / \tau_{\mathrm{s}}}$. Therefore, $\tau_{\mathrm{s}}$ denotes the sintering time scale and $n$ the sintering power law exponent. Such a law can be rigorously derived from the force laws derived in [7] for surface sintering, which yields an exponent $n=3$. For $n=1$, we obtain the well-known Frenkel law [8] for viscous sintering, for which $x / r_{\mathrm{p}} \approx \sqrt{\delta / r_{p}} \propto \sqrt{t}$. Further, if the increase of the particle radius due to sintering would be taken into account, we obtain the modified Frenkel law derived in [9]. 


\section{Simulation setup}

We model the sintering and indentation process with discrete particle simulations, using a small cubic domain of $60 \mu \mathrm{m}$, with a flat base wall and periodic boundary conditions in both width and length. We introduce 2178 particles of radii $1.35 \mu \mathrm{m}<r_{\mathrm{p}}<1.65 \mu \mathrm{m}$, producing a particle packing about 5 particle diameters thick (see Figure 2). To simulate sintering in a reasonable amount of time steps, the collision time is scaled up to $t_{c}=5 \mathrm{~ms}$, which is still several orders of magnitude smaller than the time scales of gravity, sintering, and indentation, and thus has little effect on the results. The packing is sintered using a sintering time scale of $\tau_{\mathrm{s}}=666 \mathrm{~s}$ and $n=1$ (thus assuming viscous sintering) to match the experimental results. For brevity, quantitative, more detailed results from numerical indentation tests will be presented in a later paper.

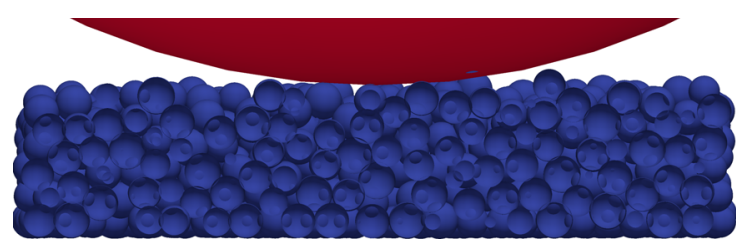

Figure 2. Vertical cut through centre of simulated sample during indentation.

\section{Results and Discussion}

\subsection{Mechanical properties}

After sintering of the PS particles samples under varying temperature-, size- and time-conditions, different densifications of the powder layer are obtained. It was found that the reduced elastic modulus $\left(E_{\mathrm{red}}\right)$ of each sintered film increased by a factor of max. 6 for sintering temperatures above $T_{\mathrm{g}}$ as the sintering time increased from $20 \mathrm{~s}$ to $180 \mathrm{~s}$. $E_{\text {red }}$ depends on the porosity of the film system.

According to Mazur et al. [10], particles smaller than a certain limiting radius are predicted to sinter to uniform density regardless of Newtonian viscosity. Consequently, the contact area initially grows much faster for particles with $r_{\mathrm{p}}<r_{\text {crit }}$ than predicted by the classical sintering models $[8,9]$, which neglect the contribution of surface forces as well as the resultant plastic and elastic contact deformation in the early stages of sintering. Assuming an initial packing fraction of $58 \%$, a critical particle radius $\left(r_{\text {crit }}\right)$ of $1.055 \mu \mathrm{m}$ for sintering of PS particles is obtained. Figure 3 shows the $E_{\text {red }}$ plotted against sintering temperatures for PS particle layers with varying radii from $0.25 \mu \mathrm{m}$ to $2 \mu \mathrm{m}$. Particles with $r_{\mathrm{p}}<r_{\text {crit }}$ show a slightly higher $E_{\text {red }}$ compared to larger particles even for temperature below $T_{\mathrm{g}}$. This hints to an additional contribution of surface force and contact deformation, which leads to faster sintering in the initial stage of sintering. Moreover, Figure 3 shows a scaling of $E_{\text {red }}$ with particle size. A transition from sintering dominated by contact deformation for $r_{\mathrm{p}}<r_{\text {crit }}$ due to surface forces and faster sintering to sintering dominated by viscous flow for $r_{\mathrm{p}}>r_{\text {crit }}$ is observed. Consequently, larger particles require higher sintering temperatures and times to show the same mechanical properties as smaller particles.

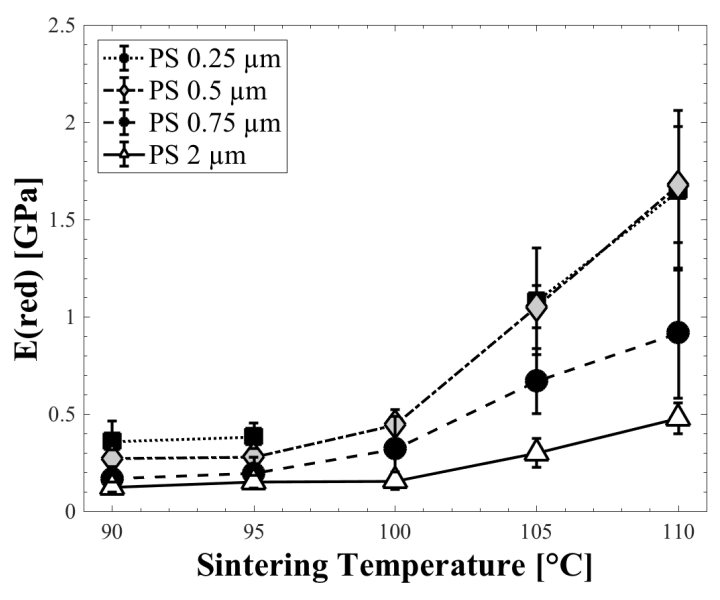

Figure 3. Mechanical properties of PS multilayers with particle radii of $0.25-2 \mu \mathrm{m}$ sintered for $60 \mathrm{~s}$. $E_{\text {red }}$ increases with the densification of the porous solids $\left(T>T_{\mathrm{g}}\right)$.

Particles with radii close to $r_{\text {crit }}\left(r_{\mathrm{p}}=0.75 \mu \mathrm{m}\right)$ seem to show both mechanisms. The contribution of the sintering kinetics to the mechanical properties of the film system is therefore more complex and does not conform to a simple scaling with particle size.

In addition to the nanoindentation results, in-situ realtime topography deformation within the sintered particle layers during nanoindentation was studied with the help of confocal microscopy. Cross-sectional xz-plane LSCM images for (a) not sintered films and films sintered at (b) $90^{\circ} \mathrm{C}$, (c) $100^{\circ} \mathrm{C}$ and (d) $110^{\circ} \mathrm{C}$ for $60 \mathrm{~s}$ at maximum indentation depth are shown in Figure 4.

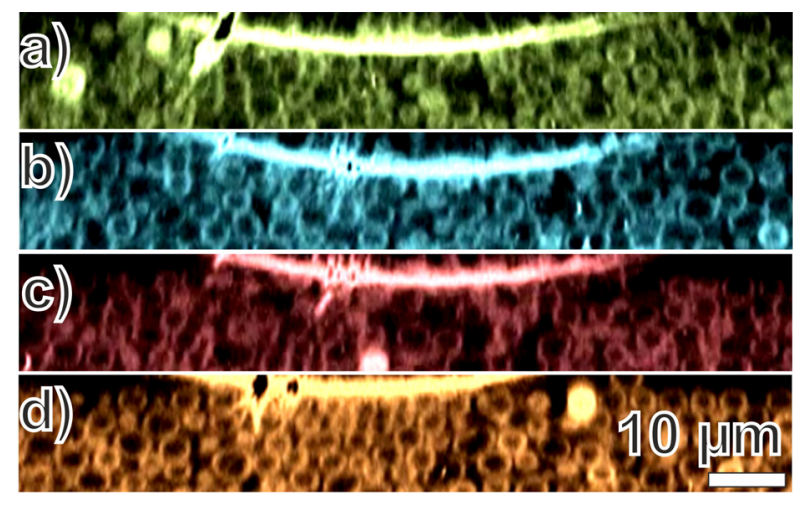

Figure 4. LSCM image (xz plane) during indentation with a spherical tip for a) not sintered PS multilayer film, b) sintered at $90^{\circ} \mathrm{C}$ for $60 \mathrm{~s}, \mathrm{c}$ ) sintered at $100^{\circ} \mathrm{C}$ for $60 \mathrm{~s}$, and d) sintered at $110^{\circ} \mathrm{C}$ for $60 \mathrm{~s}$. The maximum indentation decreases from a) to d) with increasing stiffness of the sample.

While the displacement of $5 \mu \mathrm{m}$ is reached without exceeding the limited force of the nanoindenter in case of Figure $4 \mathrm{a}$ and $4 \mathrm{~b}$, the samples above $T_{\mathrm{g}}$ (Figure $4 \mathrm{c}$ and $4 \mathrm{~d}$ ) 
show a reduced indentation depth due to a higher $E_{\text {red }}$ of the porous film system. A deformed region is observed after indentation for samples in Figure 4a-c. The sample sintered at a temperature of $110^{\circ} \mathrm{C}$ has a nearly fully elastic response. Such behavior can be attributed to a strong interconnection of the particles based on sintering necks.

\subsection{Sinter kinetics}

A representative plot of the time-dependent increase of the sintering neck radius $x$ at $110^{\circ} \mathrm{C}$ for PS particles with radius of $1.5 \mu \mathrm{m}$ is shown in Figure 5. The experimental results obtained with $3 \mathrm{D}$ reconstruction show a higher growth rate in the early stages of sintering compared to the prediction of the classical sinter models of Frenkel [8] and the modified Frenkel model [9], using a time scale of $\tau_{\mathrm{s}}=666 \mathrm{~s}$ to match the experimental observations. Simulation of viscous sintering $\left(n=1, \tau_{\mathrm{s}}=666 \mathrm{~s}\right)$ agree well with the Frenkel model, up to the point where many overlaps approach the maximum plastic overlap $(t>120 \mathrm{~s})$, while simulations using the surface sintering model $(n=$ $3, \tau_{\mathrm{s}}=110 \mathrm{~h}$ ) overpredict the early-stage sintering rate. This indicates that viscous sintering is aided by an additional contribution of surface forces to the sintering process.

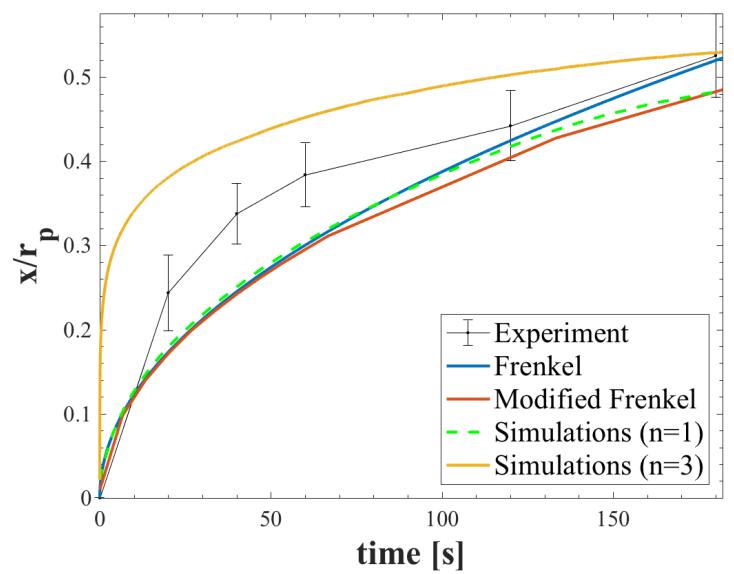

Figure 5. Sintering kinetic of PS particle multilayer $\left(r_{\mathrm{p}}=\right.$ $1.5 \mu \mathrm{m})$ at $110^{\circ} \mathrm{C}$ compared with Frenkel model (blue line), modified Frenkel model (red line), and simulations of viscous sintering $(n=1$, green line) and surface sintering $(n=3$, orange line).

\section{Conclusion}

Temperature-, time- and size-dependent sintering kinetics and mechanical properties of initial stage sintering of thin PS particle layers were investigated by utilizing 3D tomography (FIB/SEM), nanoindentation and confocal microscopy. Our experimental results show that the sintering kinetics and mechanical properties of particles with $r_{\mathrm{p}}<1 \mu \mathrm{m}$ are dominated by contact deformation due to surface forces while sintering of larger particles is characterized by viscous flow as the dominant mechanism. Consequently, larger particles require higher sintering temperatures and times to reach the same extent of sintering. To calibrate a temperature- and pressure-dependent sintering model that includes the contribution of surface forces, we use the elasto-plastic model of Luding [6], and model sintering by introducing a rate of change for the permanent, plastic deformation at high temperatures. The contact model can simulate both contact sintering as well as compression, i.e., elastic repulsion, allowing the simulation of sintering and indentation tests in a single simulation framework. The preliminary simulation results shown here are for purely viscous and surface sintering, which agree well with the theoretical predictions, but under-, respectively overpredict, the experimental results. A model taking into account both surface forces and viscous sintering, as well as the increase in particle radius due to sintering may be able to fully explain the experimental observations.

\section{Acknowledgments}

The authors would like to thank the German Research Foundation (DFG) for financial support. This work is carried out within the framework of the Key Research Program (SPP 1486 PiKo "Particles in Contact") grants LU $450 \backslash 10$ and KA $1724 \backslash 1$. We thank Laurent Gilson for his help in confocal microscopy measurements and Nina Hoinkis for sample preparation. The numerical solutions of the contact models in this paper were carried out using the open-source code MercuryDPM (mercurydpm.org).

\section{References}

[1] E.N. Antonov, V.N. Bagratashvili, M.J. Whitaker, J.J.A. Barry, K.M. Shakesheff, A.N. Konovalov, V.K. Popov, S.M. Howdle, Advanced Mat. 17, 327 (2005)

[2] R. Landers, U. Hubner, R. Schmelzeisen, R. Mulhaupt, Biomaterials 23, 4437 (2002)

[3] L. Zhang, M. D’Acunzi, M. Kappl, G.K. Auernhammer, D. Vollmer, C.M. van Kats, A. van Blaaderen, Langmuir 25, 2711 (2009)

[4] A.C. Fischer-Cripps, Vacuum 58, 569 (2000)

[5] S. Luding, Granular Matter 10, 235 (2008)

[6] S. Luding, K. Manetsberger, J. Müllers, Journal of the Mechanics and Physics of Solids 53, 455 (2005)

[7] R. Besler, M. Rossetti da Silva, J.J. Rosario, M. Dosta, S. Heinrich, R. Janssen, Journal of the American Ceramic Society 98, 3496 (2015)

[8] J. Frenkel, Journal of Physics 9, 385 (1945)

[9] O. Pokluda, C.T. Bellehumeur, J. Vlachopoulos, AICHE Journal 43, 3253 (1997)

[10] S. Mazur, R. Beckerbauer, J. Buckholz, Langmuir 13, 4287 (1997) 